\title{
Compartmentalized, multiphasic nanocolloids with potential applications in drug delivery and biomedical imaging
}

\author{
K.-H. Roh', M. Yoshida², J. Lahann ${ }^{1-3}$
}

\begin{abstract}
Nanoparticles are excellent candidates for drug delivery or biomedical imaging, because they often exhibit superb tuneability of critical properties, such as size, surface characteristics, degradation rate, and therefore drug release rates. We have recently developed a route towards fabrication of sub-micron particles that relies on electrohydrodynamic co-jetting. In this process, fluid manipulation in an electrical field is used to fabricate large quantities of multi-compartment particles, where individual compartments can be indepen-
\end{abstract}

dently loaded with different drugs or selectively surface-modified. In this contribution, aspects of multifunctional particles for biomedical applications are reviewed and a specific focus is given to recent progress with compartmentalized, multiphasic nanocolloids in our laboratory.

Keywords: Electrified jetting; electrospinning; nanoparticle; nanocolloid; multifunctional; drug delivery; biomedical imaging.

\section{Introduction}

Nanoparticles carry potential for use in drug delivery or as imaging probes because of their controllable sizes, surface characteristics, and degradation rates. By simply changing materials and formulation processes, a wide variety of characteristics can be achieved [1-7]. This has allowed for the use of nanoparticles in a wide variety of biomedical applications ranging from drug delivery to blood pool imaging. Nanoparticles have been proposed as circulating reservoirs for drugs that are associated with short circulation times (e.g. proteins and peptides) and targeted to specific tissues to minimize dose and toxic side effects [8-11]. As such, clearance of nanoparticles by macrophages and Kupffer cells of the liver upon introduction into the circulation poses a limitation to effective use of nanoparticles in medicine [12]. Efforts to render particles less prone to clearance and improve long-circulation properties include particle surface modifications as well as adsorption of particles to circulating erythrocytes [13-15]. While passive targeting strategies take advantage of pathophysiological or anatomical properties of the human body, active strategies involve selective affinity of the drug construct to recognize a specific cell, tissue, or organ. In this approach, a targeting moiety, such as an antibody, a peptide, a ligand, or an aptamer, is conjugated with the drug itself or with a nanosystem loaded with the therapeutic agent.

The vision that nanoparticles could essentially act as functional components in novel device generations, which "magically" maneuver themselves within the human body, has resulted in a new euphoria in nanomedicine. This vision has already fueled significant advances in the synthesis of a multitude of nano-objects with different properties, compositions, and structures, majority of which is still compositionally homogeneous. Nevertheless, multi-phasic nanosystems seem more feasible than ever [16]. To date, several approaches

\footnotetext{
1 Macromolecular Science and Engineering, University of Michigan

2 Chemical Engineering, University of Michigan

3 Materials Science and Engineering, University of Michigan
}

for the preparation of Janus particles using microcontact printing, partial masking, microfluidic co-flow, and selective deposition have been reported [17-23]. In such nanomaterials, the anisotropic distribution of the two or more different chemical species becomes another governing rule. We work under the premise, that a nanosystem is a material with unique properties that has subcellular dimensions and sizes below 1000 $\mathrm{nm}$, such as polymer-based nanoparticles and nanocolloids, liposomes, polysomes, micelles, or dendrimer-drug conjugates [24].

While effect of size, surface chemistry, and polymer composition on particle behavior has been studied in great detail, it is only recently that fabrication methods for non-spherical particles has enabled study of role of particle shape in applications including drug delivery [25]. Using particles of various shapes and aspect ratios, it was shown that the fate of particles with respect to phagocytosis was determined by the local shape at the point of contact between the phagocyte and the particles [26]. With increased command of fabrication methods to control various particle parameters and their fate in vivo, production of highly tailored systems via combination of these approaches becomes possible, and presents a unique opportunity for drug delivery.

In the following discussion, we will describe aspects of compartmentalized, multiphasic nanocolloids in our laboratory recently published in a series of journals [27-30].

\section{Preparation and characterization of biphasic nanocolloids}

Our approach towards biphasic nanocolloids differs fundamentally from the previously introduced methods in that it takes advantage of polymer solutions, specifically solutions of FDA-approved polymers. Using electrified polymer jets to create an anisotropic materials distribution in nanoparticles, we have developed an approach towards biphasic nanocarriers that was termed "electrohydrodynamic co-jetting" or "electrified co-jetting". Electrified jetting is a process to generate liquid jets by use of electrostatic forces [31]. Electrohydrody- 


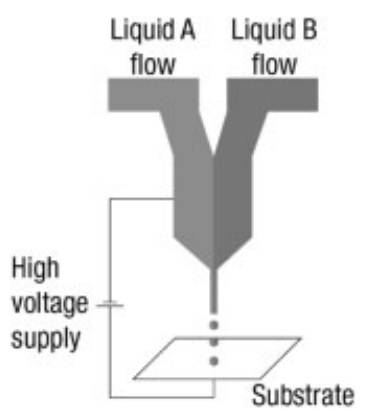

Figure 1. Schematic description of the electrified co-jetting process used for the preparation of multicompartment nanocolloids. (taken from Roh et al. Nature Materials 2005 [27])

namics has already resulted in isotropic materials made from single-phase polymer solutions, blends, and hybrid materials, as well as coaxial fibers and core/shell particles [32-38]. Nanofibers have been suggested for various biomedical applications such as for scaffolds in tissue engineering [39-41], controlled drug delivery systems [42] and enzyme-carrying biocatalyst [43]. Similarly, dual piezoelectric ejection system has been developed to produce core-shell microcapsules by taking advantage of interfacial phase separation [44].

Thus far, first promising biphasic nanocolloids have been identified using computational simulations and first prototypes of biphasic particles have been prepared [27]. In that initial work, side-by-side dual jetting capillaries [45, 46] were utilized to successfully produce a liquid cone and a jet with an anisotropic material distribution (Figure 1). With practical applications in biomedical field in mind, two jetting solutions were designed to be made of a mixture
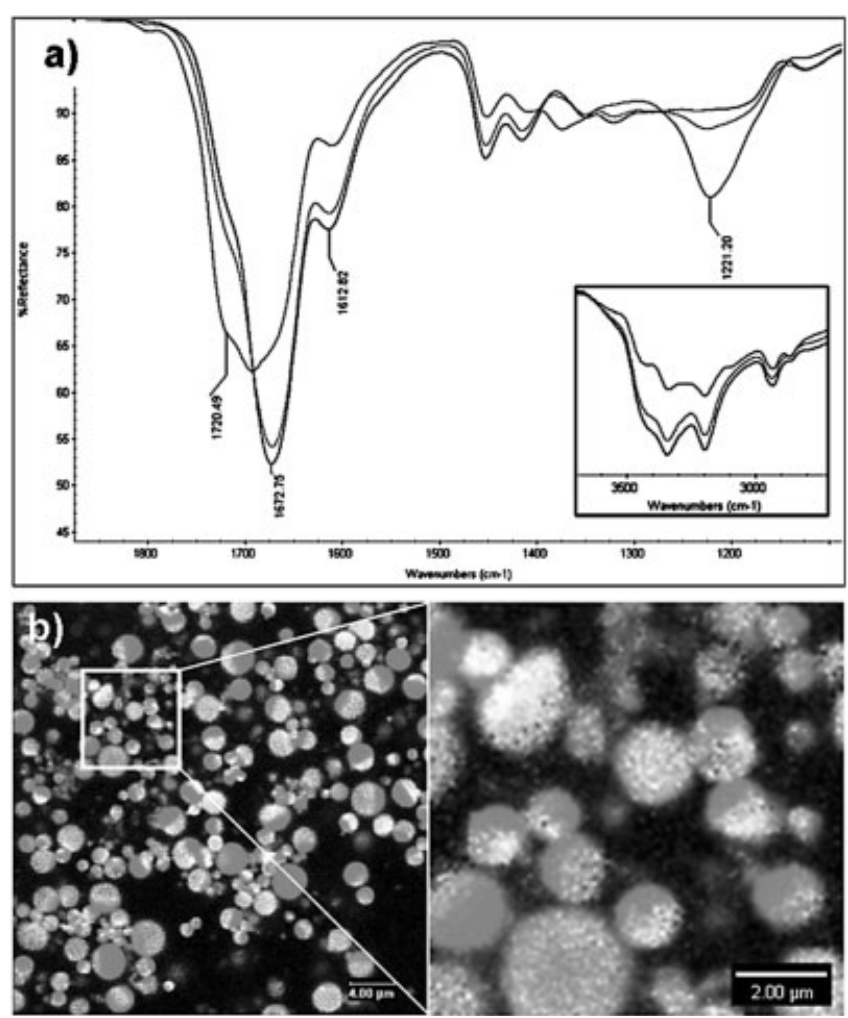

Figure 2. Stabilization of biphasic PAAm nanoparticles. a. FTIR spectra of biphasic nanocarriers before (blue) and after (green and red, 1 hour and 12 hours of reation respectively) thermal imidization; b. confocal images of particles stored and imaged in water, variable magnification, particles are between 100 and several 1000 nm. (taken from Roh et al. Langmuir 2007 [28]) of poly(acrylic acid) (PAA) and poly(acrylic amide)-co-acrylic acid (PAAm-co-AA) with varying concentrations of fluorescence-labeled macromolecules [28]. Upon establishing appropriate ratios of PAA and PAAm-co-AA, nanocolloids were prepared, and then exposed to elevated temperatures to induce crosslinking. This thermal imidization was monitored by Fourier Transform Infrared (FTIR) Spectroscopy for various reaction conditions, and was found to profoundly enhance the stability of the biphasic nanocarriers in aqueous environment. While the polymer system employed herein takes advantage of commercially available polymers which are amenable to thermal crosslinking, this stabilization method may not be appropriate in cases where the particles are loaded with drugs, especially proteins and enzymes, or with other molecules that may be fragile at elevated temperatures. In such instances, polymeric systems with UV-crosslinkable moieties and appropriate initiators may be employed for photo-initiated stabilization.

Figure $2 a$ shows a series of FTIR spectra of nanocolloids prepared using polymer composition of $5 \%$ of PAAm-co-AA with $1 \%$ of PAA. All the samples were directly jetted onto a piece of gold substrate and FTIR reflectance measured after each reaction time at $175^{\circ} \mathrm{C}$. After $60 \mathrm{~min}$, the characteristic $\mathrm{C}=\mathrm{O}$ stretching band (amide I around $1673 \mathrm{~cm}^{-1}$ ) and the $\mathrm{C}$ $\mathrm{NH}$ band of amide group (amide II around $1673 \mathrm{~cm}^{-1}$ ) decreased, while bands characteristic for imide groups appeared near $1720 \mathrm{~cm}^{-1}$ and $1221 \mathrm{~cm}^{-1}$. Figure $2 b$ shows the confocal laser scanning microscope images of biphasic nanocolloids suspended in a phosphate buffered saline (PBS) after thermal imidization. These images confirm the effectiveness of thermal reaction at $175^{\circ} \mathrm{C}$ for $60 \mathrm{~min}$ to stabilize the biphasic nanocolloids. In addition, as observed from individual phases and the overlay of the two phases, the fluorescence-labeled dextrans incorporated in each phase showed little interfacial diffusion or mixing after thermal reaction, even after storage in water for several weeks. Moreover, biphasic nanocolloids composed of $5 \%$ PAAm-co-AA and $0.5 \%$ of PAA suspended in PBS maintained their biphasic characteristic during the 2week observation period used in this study.

In biomedical applications, biocompatibility of these nanocolloids is of importance. Preliminary biocompatibility of biphasic nanocarriers composed of PAA and PAAm was evaluated using Human Umbilical Vein Endothelial Cells (HUVECs) as a model system. Confluent cells in cultured in serum-containing media were exposed to biphasic nanocarriers for indicated duration and cell viability assessed by trypan blue exclusion [29]. While this polymer system was not biodegradable, the resulting biphasic nanocolloids did not cause significant cell death, as determined by trypan blue exclusion, for up to $48 \mathrm{hrs}$ after addition of up to $1 \mathrm{mg} / \mathrm{ml}$ biphasic nanocarrier suspension (Figure 3). Further studies are underway to more thoroughly investigate the effect of biphasic nanocolloids on cells.

\section{Preparation and characterization of triphasic nanocolloids}

In a recent advancement, we have succeeded in preparation and initial characterization of triphasic nanocolloids [30]. Using the same co-jetting approach as described above, simultaneous jetting of three distinct polymer solutions was achieved with a modified nozzle with side-by-side geometry, 
a)

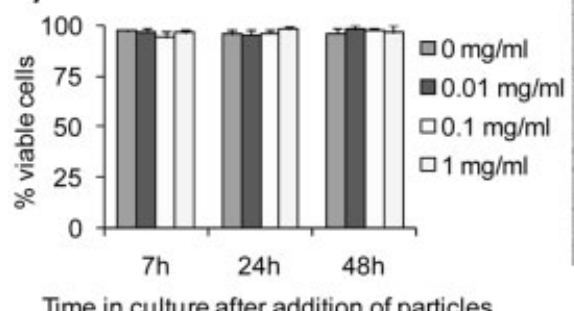

Time in culture after addition of particles b)

Figure 3. Short-term cytotoxicity test of biphasic nanoparticles. a. Time course and dose-response of biphasic nanoparticles cytotoxicity as measured by HUVEC viability by trypan blue exclusion; $b$. Phase contrast micrograph of HUVECs cultured in the presence of $0.1 \mathrm{mg} / \mathrm{ml}$ biphasic nanocolloids. (Yoshida et al. Biomaterials 2007, [29])
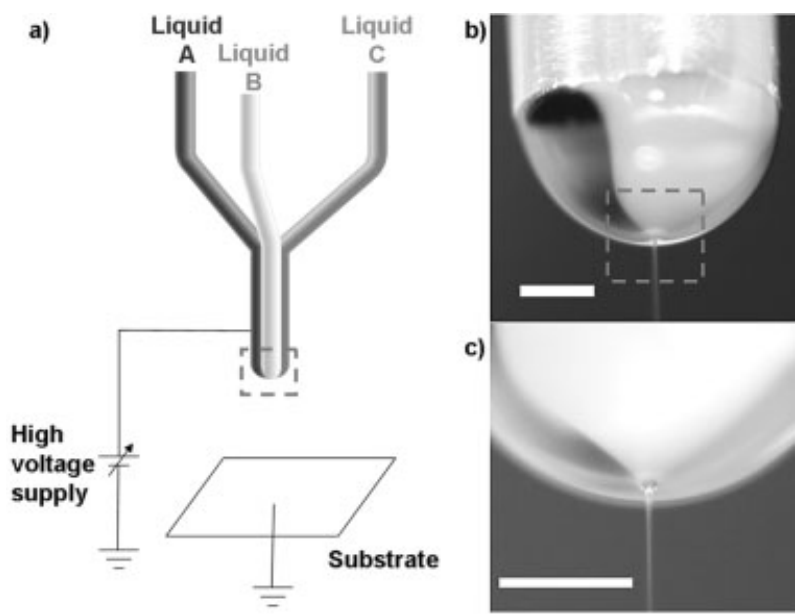

Figure 4. Production of triphasic nanocarriers. a. Electrified cojetting setup used in our experiments with triple side-by-side capillaries; b. Actual photograph of the capillary outlet region designated with dotted line in (a); c. Enlarged photograph of the jet ejection point designated with dotted line in (b). Scale bars are $500 \mu \mathrm{m}$. (taken from Roh et al. JACS 2006, [30])

as shown in Figure 4a. These nanocarriers provide three independent surfaces and three compartments for potential drug loading. Figure $4 b$ shows an image of the outlet region during the jetting process, which consists of three flows (applied electric potential is approximately $15 \mathrm{kV}$ ).

The green, red, and blue jetting solutions are composed of poly(ethylene oxide) (PEO, MW $600 \mathrm{kD}$ ) mixed with different macromolecular dyes: fluorescein-conjugated dextran (green), rhodamine B-conjugated dextran (red), and Alexa Fluor $^{\circledR}$ 647-conjugated bovine serum albumin (blue). As can be observed from Figures $3 \mathrm{~b}$ and its enlargement (Figure $4 c$ ), separation in the three phases is maintained throughout the pendant droplet. Moreover, these digital images reveal a single liquid thread maintaining the interface amongst the three phases.

These triphasic nanocolloids were characterized by confocal laser scanning microscopy (Figure 5a). As shown in the overlay of the three confocal images (Figure 5a), fluorescentlabeled macromolecular dyes loaded in each jetting liquid remained compartmentalized to form three separate phases. Although a range of different nanocolloidal architectures and interfaces co-existed, the triangular geometry of the multi-phasic nanocolloids shown in Figure 5 appeared to be predominant. In addition, scanning electron microscopy (SEM) studies of PEO-based nanocolloids revealed that these particles were linked by fine polymer threads with a diameter of the order of ten nanometers (beads-on-a-string morphology) (Figure $5 b$ ). Based on the SEM analysis, the average diameter
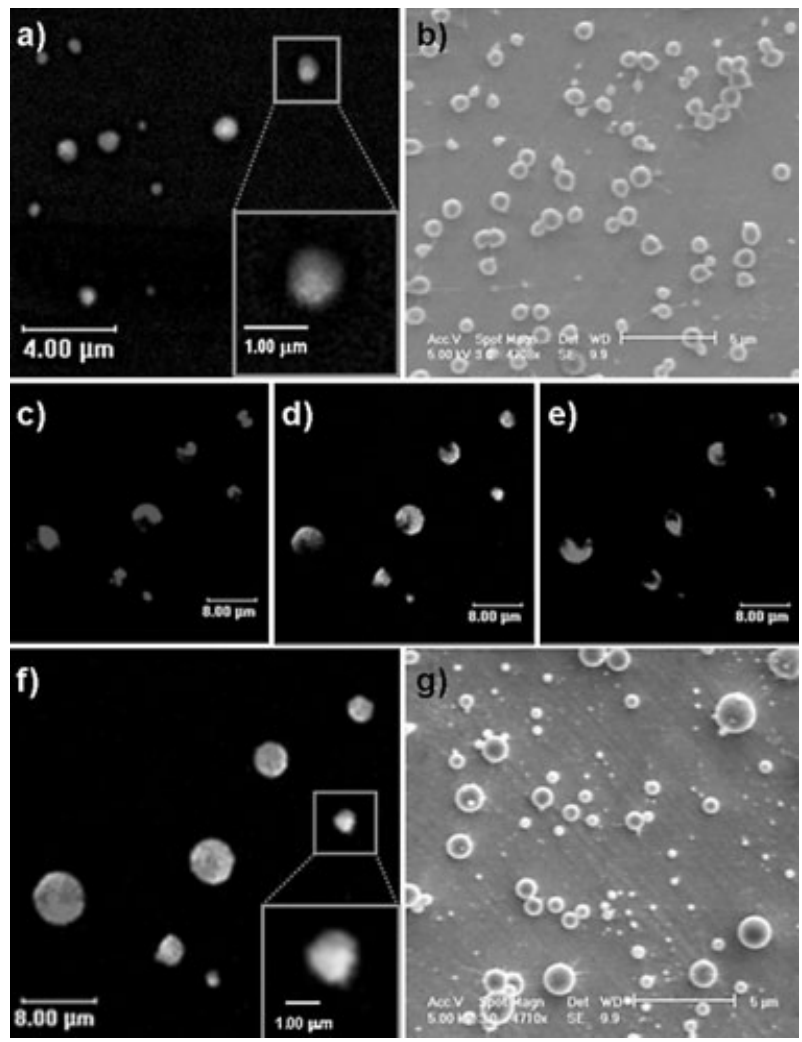

Figure 5. Confocal laser scanning micrographs and scanning electron microscopy of PEO- (a and b) and P(AAm-co-AA)-based (c-g) particles. The individual phases containing biomolecules tagged with FITC (c), Rhodamine B (d) and Alexa Fluor ${ }^{\circledR} 647$ (e) and the overlays of three phases (b and f). (taken from Roh et al. JACS 2006, [30])

of the beads was determined to be approximately $730 \mathrm{~nm}$ with a standard deviation of $260 \mathrm{~nm}$.

In principle, jetting of polymer solutions under the influence of electrohydrodynamic forces can be applied to a wide range of commodity polymers. In addition to the PEO-based system, electrified co-jetting of either PAA (MW 250 kD) or PAAm-co-AA (MW 200 kD, 10\% acrylic acid residues) solutions also produced triphasic nanocolloids . As with the biphasic particles of PAA or PAAm-co-AA, these particles were thermally crosslinked to stabilize them in aqueous environment. Figures $5 c$-e show that the fluorescent dyes were contained within each compartment, and their overlay in Figure $5 f$ confirms predominance of each dye in only one phase, indicative of tri-compartmental nanocolloids. 


\section{Future Outlook}

Future advances with the development of increasingly sophisticated drug delivery systems as well as with the design of advanced imaging probes may require the development of nanoparticles with multiple, independent compartments that can be independently loaded with different drugs. Electrohydrodynamic co-jetting provides a simple, high-throughput fabrication method towards such unique particle geometries and may play a pivotal role in the design and development of future drug delivery and imaging systems. Towards this end, future research will need to address how to reduce the often observed polydispersity of particles made by electrified jetting as well as how to extend this method to other polymer systems including polymers that can be co-jetted from non-aqueous solutions and biodegradable polymers.

\section{References}

1. C. Lemarchand, R. Gref, P. Couvreur, Eur. J. Pharm. Biopharm. 2004, 58, 327.

2. C. Lemarchand, R. Gref, C. Passirani, E. Garcion, B. Petri, R. Muller, D. Costantini, P. Couvreur, Biomaterials 2006, 27, 108.

3. P. Calvo, B. Gouritin, H. Chacun, D. Desmaele, J. D'Angelo, J. P. Noel, D. Georgin, E. Fattal, J. P. Andreux, P. Couvreur, Pharm. Res. 2001, 18, 1157.

4. Y. Aktas, M. Yemisci, K. Andrieux, R. N. Gursoy, M. J. Alonso, E. Fernandez-Megia, R. Novoa-Carballal, E. Quinoa, R. Riguera, M. F. Sargon, H. H. Celik, A. S. Demir, A. A. Hincal, T. Dalkara, Y. Capan, P. Couvreur, Bioconjugate Chem. 2005, 16, 1503.

5. P. R. Lockman, R. J. Mumper, M. A. Khan, D. D. Allen, Drug Dev. Ind. Pharm. 2002, 28, 1.

6. L. Costantino, F. Gandolfi, G. Tosi, F. Rivasi, M. A. Vandelli, F. Forni, J. Control. Release 2005, 108, 84.

7. C. Passirani, G. Barratt, J. P. Devissaguet, D. Labarre, Pharm. Res. 1998, 15, 1046

8. I. Brigger, C. Dubernet, P. Couvreur, Adv. Drug Deliver. Rev. 2002, 54, 631 .

9. L. Brannon-Peppas, J. O. Blanchette, Adv. Drug Deliver. Rev. 2004, 56, 1649.

10. J. Panyam, V. Labhasetwar, Adv. Drug Deliver. Rev. 2003, 55, 329.

11. S. M. Moghimi, A. C. Hunter, J. C. Murray, Faseb J. 2005, 19, 311.

12. J. Vasir, M. Reddy, V. Labhasetwar. Curr. Nanosci. 2005, $1,47$.

13. S. M. Moghimi, A. C. Hunter. Crit. Rev. Ther. Drug Carrier Syst. 2001, 18, 527.

14. E. Chambers, S. Mitragotri. Exp. Biol. Med. 2007, 232, 958.

15. E. Chambers, S. Mitragotri. J. Control. Release. 2004, 100, 111.

16. A. Perro, S. Reculusa, S. Ravaine, E. B. Bourgeat-Lami, E. Duguet, J. Mater. Chem. 2005, 15, 3745.
17. Z. Bao, L. Chen, M. Weldon, E. Chandross, O. Cherniavskaya, Y. Dai, J. B. H. Tok. Chem. Mater. 2002, 14, 24

18. V. N. Paunov. Langmuir 2003, 19, 7970.

19. V. N. Paunov, O. J. Cayre. Adv. Mater. 2004, 16, 788.

20. H. Takei, N. Shimizu. Langmuir 1997, 13, 1865.

21. E. Hugonnot, A. Carles, M.-H. Delville, P. Panizza, J.-P. Delville. Langmuir 2003, 19, 226.

22. O. Cayre, V. N. Paunov, O. D. Velev. Chem. Commun. 2003, 18, 2296.

23. T. Nisisako, T. Torii, T. Takahashi, Y. Takizawa. Adv. Mater 2006, 18, 1152

24. M. Ferrari, Nat. Rev. Cancer 2005, 5, 161.

25. J. A. Champion, Y. K. Katare, S. Mitragotri. J. Control. Release 2007, 121,3.

26. J. A. Champion, S. Mitragotri. Proc. Natl. Acad. Sci. U. S. A. 2006, 103, 4930 .

27. K. H. Roh, D. C. Martin, J. Lahann, Nat. Mater. 2005, 4, 759.

28. K. H. Roh, M. Yoshida, J. Lahann, Langmuir 2007, 23, 5683.

29. M. Yoshida, K. H. Roh, J. Lahann, Biomaterials 2007, 28, 2446.

30. K. H. Roh, D. C. Martin, J. Lahann, J. Am. Chem. Soc. 2006 , 128, 6796.

31. G. Taylor, Proc. R. Soc. London Ser. A 1964, 280, 383

32. J. Doshi, D. H. Reneker, J. Electrostat. 1995, 35, 151.

33. S. F. Wong, C. K. Meng, J. B. Fenn, J. Phys. Chem. 1988, 92, 546.

34. L. Larrondo, R. S. J. Manley, J. Polym. Sci. Polym. Phys. 1981, 19, 909.

35. I. G. Loscertales, A. Barrero, I. Guerrero, R. Cortijo, M. Marquez, A. M. Ganan-Calvo, Science 2002, 295, 1695.

36. D. Li, Y. N. Xia, Nano Lett. 2004, 4, 933.

37. J. H. Yu, S. V. Fridrikh, G. C. Rutledge, Adv. Mater. 2004, 16 , 1562.

38. Z. C. Sun, E. Zussman, A. L. Yarin, J. H. Wendorff, A. Greiner, Adv. Mater. 2003, 15, 1929

39. H. Yoshimoto, Y. M. Shin, H. Terai, J. P. Vacanti, Biomaterials 2003, 24, 2077.

40. W. J. Li, C. T. Laurencin, E. J. Caterson, R. S. Tuan, F. K. Ko, J. Biomed. Mater. Res. 2002, 60, 613.

41. E. D. Boland, G. E. Wnek, D. G. Simpson, K. J. Pawlowski, G L. Bowlin, J. Macromol. Sci. Pure 2001, 38, 1231.

42. E. R. Kenawy, G. L. Bowlin, K. Mansfield, J. Layman, D. G. Simpson, E. H. Sanders, G. E. Wnek, J. Control. Release 2002, 81, 57.

43. H. F. Jia, G. Y. Zhu, B. Vugrinovich, W. Kataphinan, D. H. Reneker, P. Wang, Biotechnol. Progr. 2002, 18, 1027.

44. Y. Yeo, O. A. Basaran, K. Park. J. Control. Release 2003, 93, 161.

45. S. Madhugiri, A. Dalton, J. Gutierrez, J. P. Ferraris, K. J. Balkus, J. Am. Chem. Soc. 2003, 125, 14531.

46. P. Gupta, G. L. Wilkes, Polymer 2003, 44, 6353.

Corresponding author: Prof. Jörg Lahann, Macromolecular Science and Engineering, Chemical Engineering and Materials Science and Engineering, University of Michigan, lahann@umich.edu

Received in final form: November 8, 2007

[T 245] 